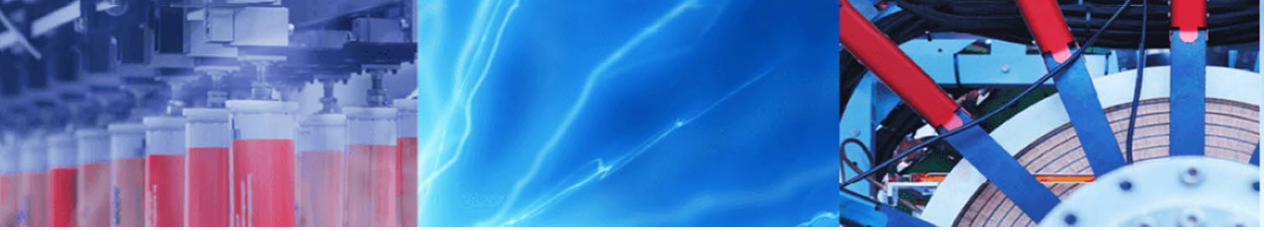

Case Study

\title{
Dynamic relationship design of knowledge co-creating cluster: traditional Japanese architectural industry
}

\author{
Akane Matsumae $^{1}$ D $\cdot$ Susumu Matsumae ${ }^{2}$ (D) Yukari Nagai $^{3}$ (D)
}

Received: 30 September 2019 / Accepted: 7 February 2020 / Published online: 18 February 2020

(c) Springer Nature Switzerland AG 2020

\begin{abstract}
Increased global mobility led by remarkable developments and the extension of public infrastructure has brought about not only the possibility of further conventional static relationship design, but also the importance of dynamic relationship design as well. Operating on the assumption that this catalytic environmental change will occur in the form of increased mobility, every type of organization — regardless of industrial sector, size, or profit status—is faced with the need to design and manage this connected society, newly dynamic environment. Considering the social environment above, this study aims to develop a methodology to design and manage innovation ecosystem which then generates sustainable and autonomously collaborative relationships as the inevitably required first phase of organization emergence. How can a dynamic relationship as a knowledge co-creating subjectivity be formed among the organizations? The authors introduced research methodology of dynamics, referring to knowledge creation theory: (1) proposing a dynamic system model simplifying research objects, (2) conducting the cases based on the proposed model, (3) performing theoretical analysis on observed phenomena, and (4) engaging in discussion on improvements, or further development of the original model based on the findings. In this study, the authors visualized the relations among co-creation, intersubjectivity, context, and boundary object in a dynamic model, and described the organizational emergence of knowledge-creating clusters using the dynamic model. This study contributes to the social shift in a contrasting approach, from the management of human well-doing for an efficient social system to the dynamic relationship design for human well-being.
\end{abstract}

Keywords Relationship design · Organization emergence · Social creativity

\section{Introduction}

\subsection{Background}

In a connected society with increased mobility represented by the term Industry 4.0, where a decentralized, self-organizing, and flexible production environment will replace the classical, centrally controlled production hierarchy [4], every type of organization is faced with the need to design and manage a newly dynamic environment. From the viewpoint of SMEs in Japan, for instance, this can be the opportunity to form the innovation ecosystem for their own, considering most of local SMEs still remain dependent on vertical relationships with larger companies and excluded from innovative opportunities, channels, and human resources under the current long-standing dominant centralized innovation ecosystem.

Thus the importance of collaboration among diverse stakeholders [28], but at the same time, the difficulties in its implementation have also been recognized. Simply gathering people from various organizations is not enough to form a basis for sustainable collaborative innovation: developing methodology to flexibly design,

Akane Matsumae, matsumae@design.kyushu-u.ac.jp | ${ }^{1}$ Kyushu University, 4-9-1 Shiobaru, Minamiku, Fukuoka, Japan. ${ }^{2}$ Saga University, 1-1 Honjo, Saga, Japan. ${ }^{3}$ Japan Advanced Institute of Science and Technology, 1-1 Asahidai, Nomi, Ishikawa, Japan. 
implement, and manage the relationships that structure a society has become a broader need.

This study focuses on the formation process of horizontal knowledge co-creating cluster generated among organizations in different contexts. To guide this study, the research question was defined: How can a dynamic relationship as a knowledge co-creating subjectivity be formed among the organizations in various contexts?

\subsection{Definition of co-creation}

The term co-creation in the research question above has not reached a common definition and the distinction among collaboration, co-creation, and co-operation has remained ambiguous $[1,24,27]$. The definitions and discussions on co-creation in existing literature indicate that the aspect of focus in a study ought to be reflected in the definition.

Therefore, for the purposes of this study, the authors define co-creation in this study as a collaboration to create something together sharing the phase of socialization among individuals, where socialization is the tacit knowledge-sharing phase among individuals referring to the SECl spiral [19].

Sharing a clear common goal among individuals is not required at the beginning of the process, but the goal appears during the process of dynamically developed cocreation. In contrast, co-operation is distinctive from cocreation because it is collaboration to achieve a clear given common goal from the beginning (Fig. 1) [15]. When a collaboration is purposed to bring a defined goal, it is efficient

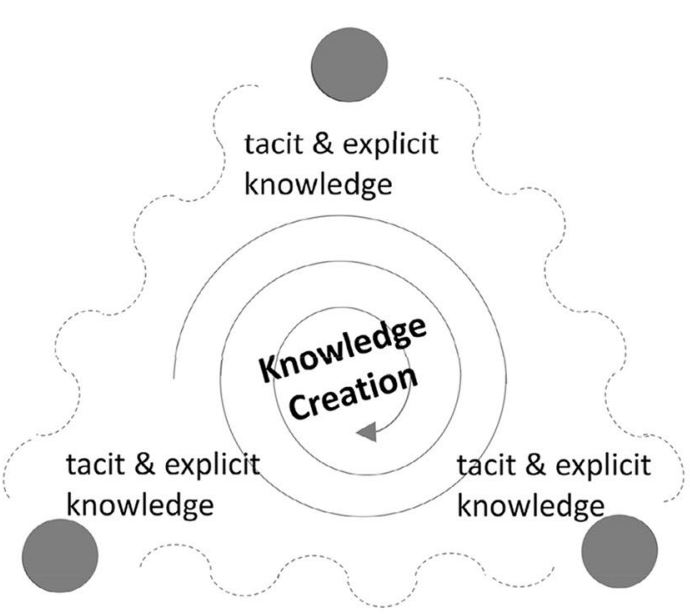

Co-creation to set and pursue a clear goal to achieve. However, this study focuses on a dynamic phase of collaboration, cocreation, where any specific goal has not emerged yet, and it is fundamentally important to generate and to sustain the co-creation phenomena itself as if it is a living organism. Konno also emphasized that setting a goal enhances efficiency but restricts dynamic and creativity for its static and fixed nature [11].

\section{Related studies}

Studies on co-creation have mainly focused on the usefulness of co-created values or on efficient collaborative processes creating these values. Few studies exist that take into consideration the fragile and dynamic nature of forming and sustaining co-creating subjectivity among individuals [12]. Studies on collaboration need to pay sufficient attention to distinguishing the fundamental differences between collaboration based on individual creativity and co-creation. In this regard, studies focused not only on the co-created value but also on the co-creating subjectivity are essential, particularly on their inner aspects as well as in reference to existing literature based on individual collaboration or creativity.

On the other hand, this perspective is also significant in terms of knowledge science. First, since knowledgeenabling conditions and the $\mathrm{SECl}$ model have mainly been developed empirically within established larger companies $[19,34]$, it should be carefully examined using evidence from experiments to expand its theoretical scope to the formation

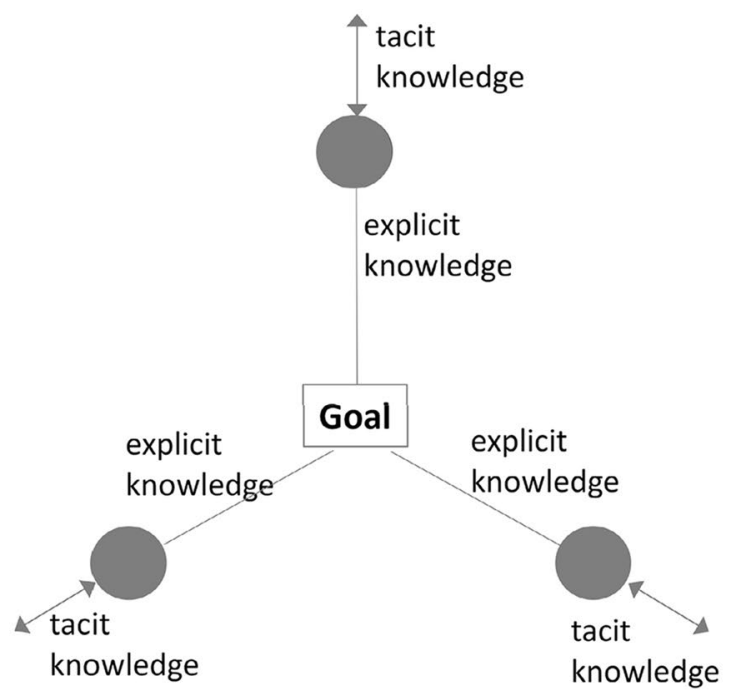

\section{Co-operation}

Fig. 1 Co-creation and co-operation [15] 
phase of interorganizational knowledge-creating entities on which we focus in this study. Little practical evidence has been demonstrated for the formation of quadruple knowledge clusters among organizations, although Nonaka and Konno have mentioned its theoretical possibility [18]. Second, as Nonaka defines organizational knowledge creation as the process of making available and amplifying the knowledge created by individuals, as well as crystallizing and connecting it to an organization's knowledge system [21], knowledge management has focused on the co-created knowledge rather than the knowledge co-creating subjectivity. This study focuses more on the co-aspect, the co-creating subjectivity.

This study takes on the challenge of elucidating a dynamic mechanism of co-creation related to the formation process of co-creating subjectivity in its gestation phase [26] and of contributing to the methodology for the design and management of flexible relationships facilitating autonomous and horizontal collaborations not only within but also among organizations. It will empirically explore the limitations of these theories-whether they can be applied during the formation phase of organizations and whether the theories apply both within and among organizations.

\section{Methodology}

In this study, we took an experimental approach in referring to literature to understand the dynamic mechanisms of co-creation in relation to the formation process of cocreating subjectivity among individuals in various contexts during the gestation phase of autonomous and horizontal collaborations. The case study was conducted through participatory observation. Our methodology is characterized by the analogy of the research methodology of dynamics, which captures interactive and integrated systems. This methodology is usually understood through four experimental approaches: (1) proposing a dynamic system model simplifying research objects, (2) conducting experiments/cases based on the proposed model, (3) performing theoretical analysis on observed phenomena during the experiments/cases, and (4) engaging in discussion on modifications, improvements, or further development of the original model based on the findings, in consideration of the possibilities of practical application.

\section{Dynamic system modeling}

\subsection{Modeling methodology}

The significance of systems modeling approaches to creativity has been argued and recognized as the theoretical foundation of the field [23]. This could be basically expanded in its course into that for co-creation, because various kinds of co-creation models have recently been proposed from which the perspective of measuring value co-creation [30]; evaluating co-creation possibilities [10]; knowledge-sharing risk management procedures [3]; understanding the consequences of co-creation among consumers, providers, and partners [32]; and the co-creation process [8].

However, most of models of co-creation, including the ones above, represent only static and/or discrete relations, which only relate to and structure factors and/or phases focusing on input and output. As discussed in the previous chapter, co-creation has a dynamic nature. Therefore, a dynamic system modeling approach can represent its fundamental nature more accurately by enabling it to describe its dynamic mechanisms as a dynamic integrated system using various factors. Several challenges in the development of dynamic systems modeling are found in the literature on creativity. For example, the systems model to justify as phenomena of creativity is analogous to the model having used to describe the process of evolution, which occurs when an individual organism produces a variation selected by the environment and transmitted to the next generation [7] and to the molecular model from behavioral perspectives [29]. The former model is based on whether creativity is worth both being included and assists in surviving in a particular field: It is incompatible to the definition of co-creativity in this study. The latter is discrete, and it cannot describe continuous and interactive behaviors of a co-creative nature.

The authors emphasize the importance of capturing co-creation mechanism as a dynamic system containing major factors, rather than focusing on a specific major factor to apply the mechanism to the design methodology for co-creation. For this purpose, a dynamic system model is proposed to represent the relationships between the formation of co-creating subjectivity and the co-creative process, based on the knowledge obtained from the experiment.

\subsection{Fundamental factors of co-creation}

In this study, the dynamic nature of co-creation is discussed from the points of view of three components: subjectivity, process, and consequence.

\subsubsection{Intersubjectivity}

Unframed subjectivities in co-creation are basically free, brittle, and dynamic enough to decimate the basis of collaboration. Therefore, it is important to understand the 
dynamics of the autonomic co-creating subjectivity, hereafter intersubjectivity, for sustainable innovation.

The concept of intersubjectivity has its origins in Husserl's phenomenology and has been developed in several different directions based on its different aspects. The domain of intersubjectivity lies beneath the empathy of the Husserl's Fifth Cartesian Meditation $[9,35]$ and the basic idea of intersubjectivity was reviewed that subjects do not constitute a world alone, but jointly, together with other subjects [6]. In the context of the subjectivity of cocreation, it has been argued by Benjamin to be intersubjectivity. From his research interest on how we actually experience the other as a separate yet connected being with whom we are acting reciprocally, he defines intersubjectivity in terms of a relationship of mutual recognition a relationship in which each person experiences the other as a like subject, another mind with which things can be experienced, yet one that has a distinct, separate center of feeling and perception [5].

The importance of intersubjectivity on which individuals share common thoughts and experiences based has been pointed to as a source of motive power for execution in knowledge co-creation [18], although it is difficult to find experimental research to support their indication in their literature because of the complexity of the phenomena of or difficulty in evaluating a human's inner state.

\subsubsection{Contexts}

In order to track a dynamic process of co-creation, the authors introduced the concept of context vector for each individual to join the project. Some contexts are described in the same dimension, while others are described in different dimensions [15]. Each individual or organization also has its own original context. Dynamic interactions among each individual's creativity, mutually affecting and altering one's context, mediated with $b a$, defined as "a shared context in motion" which can transcend time, space, and organization boundaries to create knowledge [20].

\subsubsection{Co-creativity}

Co-creativity as the inner consequence of co-creation is generated among individuals, in contrast with creativity, which is generated within an individual. This means that co-creativity is founded on a dynamic subjective basis. This point of view is especially important when we deal with either the fuzzy front-end phase in process development [25] or the gestation phase in organization development $[26,27]$, in which this study focused on for its dynamic phase in forming both its clear goal and its subjectivity.
Considering that both the essential factors of creativity, effectiveness, and novelty, can be approached through the continuous creative process, and that it is motivation which initiates and sustains the creative process [2], the most essential factor of co-creativity can be said to maintain shared motivation based on dynamic intersubjectivity among individuals, not within an established organization, to continue improving a project without a clear common goal.

Therefore, co-creativity is defined in this study as $a$ shared motivation among individuals to develop and realize their concept since it is motivation which initiates and sustains the creative process [2].

\subsubsection{Boundary object}

A boundary object is an object, which is adaptable to multiple viewpoints and robust enough to maintain identity across them [31]. In later studies, the boundary object was scaled up as an arrangement that allows different groups to work together without consensus, especially in the context of a collaboration infrastructure [12]. By setting an appropriate boundary object in the project, intersubjectivity and co-creativity can be formed where different contexts can be bundled and sharpened. [15]. Star who had advocated and developed the concept of boundary object criticized the ignorance of this dynamics in related researches and denied this major assumption inherent in collaboration studies as follows:

"Many models, in the late 1980s and continuing today, of cooperation (co-creation in this study) often began conceptually, with the idea that first consensus (a clear common goal in this study) must be reached, and the cooperation could begin. From my own fieldwork among scientists and others cooperating across disciplinary boarders, and two historical analyses of heterogeneous groups who did cooperate and did not agree at the local level, it seemed to me that the consensus model was untrue. Consensus was rarely reached, and fragile when it was, but cooperation continued, often unproblematically. The dynamic involved in this explanation is core to the notion of boundary objects" [12].

This Star's criticize against the ignorance of dynamics is especially significant in the context of this study focusing on a dynamic process to form intersubjectivity as a gestation phase of organization, comparing with the contexts of an established organization where knowledge-creating theory [19] is mainly developed.

\subsection{Inverted vortex model}

From observations of accumulated case studies, the authors have found the secondary effect of co-creation to 
form an intersubjectivity to lead autonomous knowledgecreating cluster among various stakeholders [13, 14]. Here the word secondary is used to emphasize the difference between this study and knowledge management [19] which have focused on the primary effect of co-creation, the knowledge co-created. This study tries to grasp and visualize co-creation dynamics as a phenomenon focusing on a dynamic intersubjectivity as a co-creating subjectivity in contrast to the ones to understand and manage ba for better knowledge co-creation. Furthermore, the dynamic relations among the fundamental factors of cocreation phenomena, which have been explained statically and separately, have been analogically modelized as IVM, inverted vortex model, as the result of the basic experiment (Fig. 2) [15, 16].

IVM is described as an analogy of vortex; one is the size of a boundary object to describe its reach and vorticity of the co-creation indicates how the co-creation works to each individual and contexts. The point emphasized in IVM represents expressing the dynamic mechanism of cocreation: what comes first is not a common goal or intersubjectivity among individuals, although it is necessary for these to originally appear within a certain boundary object to start co-creation. A common goal and intersubjectivity are materialized and developed alongside co-creation as individual contexts are converged.

This IVM indicates that individuals with various contexts form intersubjectivity through co-creation, as moving objects, black points in Fig. 2, converge at a deeper point along with the fluid flow of the vortex under appropriate conditions. The co-creation process and individuals' contexts interact with each other as a vortex, and the moving objects interact with each other. The moving objects are converged in the vortex, and moving objects transform, occasionally breaking the vortex.

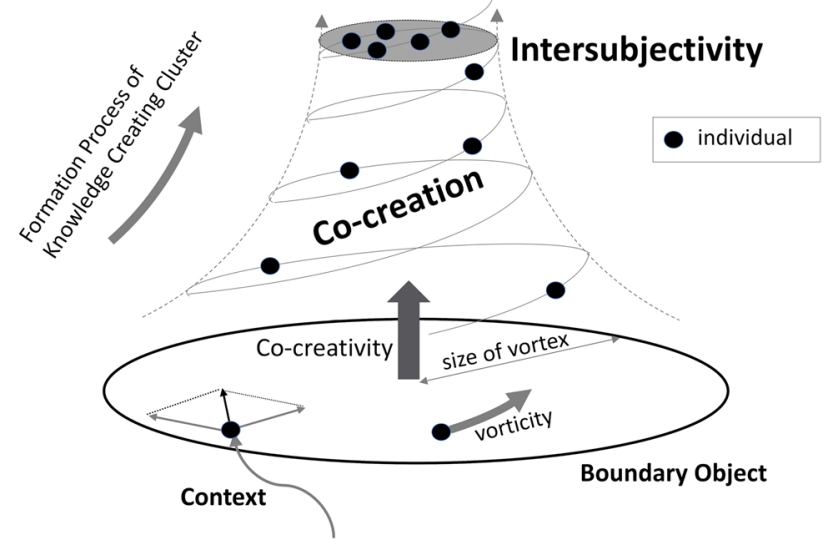

Fig. 2 Inverted vortex model $[15,16]$
The sum of the context force vector components (vorticity) that consist of bundles of context force factors with different directions and magnitudes make a whirlpool with a specific direction through the co-creation process. Intersubjectivity among the members of the project is formed as a whirlpool, a co-creation process sharing socialization, externalization, combination, and internalization. Individuals convert tacit knowledge to new tacit knowledge through shared experiences and social interactions.

From a practical standpoint, when the speed and power of a whirlpool get higher, the inner part of the whirlpool becomes more dense and more compact. Likewise, according to the model, when co-creation gets stronger, intersubjectivity gets more solid. This is how knowledgecreating clusters form through the co-creation process.

The factors of IVM seem to parallel Amabile's factors of motivation. Amabile indicated that motivation includes two factors: the individual's baseline attitude toward the task and the individual's perceptions of his or her reasons for undertaking the task. Baseline attitude is explained as the degree to which it matches his or her existing preferences and interests, and perceptions are proposed that depend largely on external social and environmental factors [2]. The boundary object in IVM has a similar role to Amabile's baseline attitude since the boundary object indicates the boundary of an individual's existing preference and interests. Likewise, context in IVM works similarly to perception since it leads to an individual's creativity.

The IVM visualizes the mechanism of the co-creation process and explains the whole fundamental mechanism in a diagram, rather than through specific factors, as in previous models.

This model was developed based on the result of experiments [16] and improved through discussions with experienced practitioners who lead decentralized knowledge clusters. These included both locally rooted contexts and the global context, namely the Knowledge Innovation Community of the European Institute of Innovation and Technology. Thus, this analogical model has in fact succeeded in applying a flat form that enables practitioners to describe, discuss, review, and explain the co-creation dynamic process between/within the knowledge clusters, absorbing practical viewpoints and reasonable suggestions of local and international practitioners.

\section{Case study}

\subsection{Problems of the case}

Excessive pursuit of economic efficiency in Japan has devastated the traditional architectural industry. Traditional Japanese architecture (hereafter TJA)'s holistic but 
economically less efficient production system has been decimated. Moreover, Japanese architectural regulations based on engineering knowledge in the late modern period underestimated the seismic performance of TJA. However, regional SMEs, which were to innovate and carry on TJA for future, seldom had sufficient resources or social credibility to deal with these problems by themselves.

Considering the above, the authors designed and managed this project expecting: to accumulate reliable data to prove the engineering rationale and effectiveness of TJA structure, to organize an autonomous and sustainable knowledge-creating cluster in horizontal relationships, to bring intellectual commitment to workers back in the production field both to empower them, and to offer real field to universities.

\subsection{Formation process of knowledge-creating cluster}

\subsubsection{Formation of the core}

In 2002, an e-mail was posted to the regional mailing list, a group of various occupations including academia, industry, government and media. In the e-mail, an ecologist asked help to hold a 1-year series of seminars by a well-known structural engineer specializing in the field of TJA. Three mailing list group members from architectural industry who had spontaneously felt interested in her e-mail got together to discuss how to host the one and started its preparation as core members (Fig. 3).

\subsubsection{Diverse participants}

A regional association of carpenters decided to host the seminars. Since the attitude of the host association was accepting, the seminar and following activities were managed not only for a specific industrial community but welcomed diverse organizations and individuals interested in TJA. This diverse group included carpenters, designers, managers, and local government officers, all of whom usually work in different culture and contexts. Thus, an agglomeration of diverse organizations gradually developed along with the series of seminars.

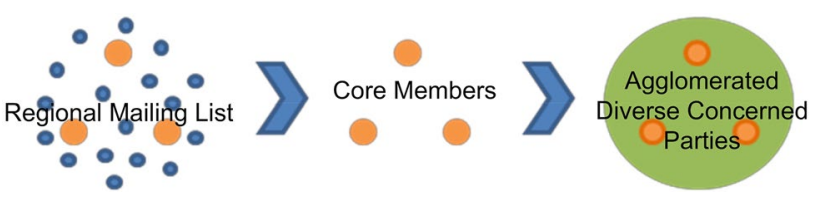

Fig. 3 How diverse concerned parties had agglomerated

\subsubsection{Increase on both interest and confusion among participants}

The seminars were just a knowledge transfer from the lecturer to the participants. However, participants increased both their interest and their confusion. The confusion was caused by the dichotomy between the outdated regulations participants had been using, and what they were being taught at the seminars based on the latest engineering. To alleviate this confusion, in December 2002 , core members planned and prepared the first primitive structural experiments.

\subsubsection{Outburst of autonomy}

In May 2003, carpenters built several types of scaleddown units and the first structural experiment was realized. They captured the difference in collapse behaviors between modern units and traditional ones, although they were not able to examine some of the typical seismic elements of TJA structure in these downscaled models.

By this time, the confusion among participants (Sect. 5.2.3) had been cleared and was replaced by the desire to pursue real knowledge based on their own professionalism. During and after this experiment, an engineer from the public research institute joined the activity. They could not help moving toward the next step.

\subsubsection{Involvement of university to evolve the project}

Soon after the first experiment, core members visited a professor to ask his cooperation for the full-scale structural experiment. In July 2003, they carried out the first full-scale seismic structural experiment. It is said to be the first full-fledged experiment in Japan ever led by carpenters in the field, according to an authorized practitioner, Kazuma Masuda. An increased number of regional architects and local government officers, and mass media came to observe this experiment and some of them joined the community.

This experiment demonstrated the obvious fact that TJA has different but effective seismic mechanisms from modern architecture. TJA does not rely only on rigidity but on the balance of rigidity and flexibility. Experiments confirmed the engineering effectiveness of TJA structure and engendered their desire to continue the experiments. They hoped to gather reliable engineering data to support the rescinding of unreasonable regulations and to improve TJA structures. Thus, their community formed and shared a common mission among diverse organizations. 


\subsubsection{Sustainable growth of the project}

Even after the series of seminars finished in 2003, their collaboration continued to hold experiments and seminars to exchange and create knowledge on TJA. Various professionals related to this mission were involved, and various funds were drawn from them. Researchers from other universities and related fields raised the quality of the project.

The flexible attitude and the passion of the missionbased community naturally led to the expansion of the range and the depth of the collaboration. The more contexts the community contained, the more important became the role of core members as the catalyst among various participants.

\subsubsection{Expansion of the project to nationwide organization}

Concomitantly, the same set of seminars was launched all over Japan and these regional seminars linked together to share knowledge. This nationwide network was incorporated as a nonprofit organization in 2005. More than 400 carpenters, architectural engineers, forestry industry workers, researchers, and citizens sharing the mission joined.

Even after the foundation of this nationwide organization, substantial activities continued in each region.

\subsection{Co-creation of the case}

In this case, co-creation (Sect. 1.2) was realized among diverse participants by sharing structural experiments. Structural experiments were developed by repeating the following basic process; each of the participants acquired tacit knowledge directly by observing structural experiment (socialization), and discussed the next experiment together based on analysis of the data and phenomena observed from each of their perspectives.

Contrary to the series of seminars (Sect. 5.2.2), which just transfers explicit knowledge from the lecturer to the audience, those who were involved in structural experiments recognized themselves as members concerned with the project. In other words, intersubjectivity was formed among the participants along with knowledge co-creation. A common context was generated based on intersubjectivity and autonomous actions within the common context arose spontaneously.

\subsection{Contexts of the case}

\subsubsection{Contexts of stakeholders in industry}

The representative contexts, in other words, motivations of various stakeholders from TJA industry, were originally to acquire education, research and channel for innovation in addition to a stamp of credibility.

First, stakeholders from TJA industry were educated and empowered with engineering knowledge of TJA through this collaboration. This educational function gave the younger participants, or brought back to the senior ones, not only engineering knowledge, but also an independent mindset, confidence, and self-esteem. These educated and empowered individuals gradually began to try to leave the fixed vertical industrial system, which had been treating them as just mindless laboring bodies. Second, the industry side got competent researchers and research facilities to solve their fundamental questions. With this collaboration, they finally got the opportunity to support their hypothesis with convincing data. As if this was water absorbed into dry land, they were eager to further research and development to take responsibility for their own profession. Third, a number of channels for innovation were opened; ones to academia, media, policy, information, grants, competent job seekers, core customers, and fellow engineers or cross-industrial partners to rejuvenate their work together. Last, the data and activities under the university-industry collaboration lend a social credibility to SMEs. This credibility created remarkable social impact and also differentiated involved SME's businesses from others.

Thus, this collaboration provided a holistic solution to the serious and deep-rooted problems TJA industry faced. It enabled the SMEs involved to break free from the fixed vertical industrial system in terminal condition, and to restructure it into a flexible horizontal industrial system.

\subsubsection{Contexts of university}

It is actually difficult to maintain fruitful university-industry collaboration without having an association with the research or educational contexts of a university. In this case, the collaboration expanded even to other research groups in the same university.

First, both the university and industry were able to share the same research interest. The industrial side willingly offered the field of research and education as well as practical knowledge and skills. Second, students learned and studied with actual production fields and people. Students were highly motivated and deeply interested in their research theme, knowing its necessity. 


\subsubsection{Common contexts shared among the stakeholders}

The series of experiments confirmed the efficacy of TJA structures in absorbing earthquake energy with its flexibility and even demonstrated that too much rigidity which lacks the balance of rigidity and flexibility does harm the entire structure. This is contrary to the opinion which led to unreasonable regulations. The contrastive differences between traditional and modern architectural structures were clearly observed concerning characteristic seismic performance and the collapse mechanism.

They experimented not only to accumulate data on seismic performance, but also to continue research and development on several types of innovative units in harmony with TJA. Some of the results were implemented into the renovation of TJA.

\subsection{Co-creativity of the case}

Co-creativity defined as a shared motivation among individuals to develop and realize their concept (Sect. 4.2.3) was observed in this case as the continuous collaboration to hold experiments and seminars to exchange and create knowledge on TJA even after the series of seminars finished in 2003. Various professionals related to this mission were involved and various funds were drawn from them. These are the obvious signs where the emerged co-creation phenomenon is sustained and moving forward with co-creativity. The flexible attitude and the passion of the mission-based community naturally led to the expansion of the range and the depth of the collaboration (Sect. 5.2.6).

\subsection{Boundary object of the case}

In this case, structural experiment was chosen as a main boundary object (Sect. 4.2.4). The size of boundary object was large enough to bundle various organizations and individuals related to TJA. The vorticity of the boundary object was strong and stable enough to keep involving participants in various contexts and to sharpen activity toward their goal. The component of context vector of each participant toward the center of the boundary object strengthened, since experiments on a life-threatening seismic problem aroused their professionalism and the strong desire for unproved true knowledge.

TJA could also be a boundary object, with a scale larger than a structural experiment but with a vorticity too weak to form a knowledge-creating cluster. The series of seminars was also too small and too weak to function as a boundary object.

\section{SN Applied Sciences}

\section{Results and discussion}

Followings are the results and discussions of the case to answer the research question defined in: How can a dynamic relationship as a knowledge co-creating subjectivity be formed among the organizations in various contexts? (Sect. 1.1).

\subsection{Phases of knowledge-creating cluster formation of the case}

The authors captured the five phases of the formation process of knowledge-creating clusters, in other words, intersubjectivity as a subject of co-creation: agglomeration of diverse concerned parties (Phase 1, hereafter P1), introduction of boundary objects (P2), activation of autonomous knowledge creation (P3), formation of a common context through co-creation (P4), and stabilization (P5).

Agglomeration of diverse concerned parties is a necessity but is just the starting point to form a knowledge-creating cluster (P1). Since each stakeholder stands in various contexts, the relationship-building mechanism must be inclusive enough to cover various contexts. The boundary object functions as this mechanism (P2). After stakeholders are bundled enough by a boundary object, it is necessary to activate them for autonomous dynamic knowledge creation. The internal motivation of each stakeholder arising from autonomous creative activity is the engine that sustains the cluster (P3). Next, particular components of various contexts among stakeholders gradually converge into a common context and intersubjectivity among stakeholders is formed through the process of knowledge cocreation (P4). Although flexibility is a great advantage in the autonomous creative phase, a minimum framework is then introduced to stabilize the very fragile intersubjectivity when stakeholders have reached a certain convergence of autonomous common context (P5).

In the five phases of formation process of knowledge co-creating clusters, knowledge creation happens mainly in P4. From P1 to P3 are regarded as the preparation for knowledge creation and $\mathrm{P} 5$ is the transitional process to bridge from the dynamic stage to the static stage. In other words, to foster conditions of knowledge creation and knowledge creation itself is the major function of formation process of knowledge co-creating clusters. Therefore, the formation process of knowledge clusters and IVM corresponds to enabling conditions for organizational knowledge creation; intention, autonomy, fluctuation and creative chaos, redundancy, and requisite variety $[19,34]$.

P1 corresponds to requisite variety. The size of the boundary object to be introduced in P2 must be large 
enough to include requisite variety but must be small enough to converge intention. It is also effective to separate an initial boundary object or even to introduce a complementing boundary object to enhance autonomy during the subsequent formation process from $\mathrm{P} 3$ to $P 5$. The vortex corresponds to knowledge co-creation observed in P3 and P4. The vorticity of the boundary object should be kept controlled, observing the dynamic balance of intention, autonomy, creative chaos, and redundancy in each phase. The stronger the vorticity, the sharper a common intention appears. In contrast, the weaker the vorticity, the more space is available for redundancy. Creative chaos and autonomy are both activated and limited by vorticity.

Intention is explained in knowledge creation theory as follows: the knowledge spiral is driven by organizational intention, which is defined as an organization's aspiration to its goals [17]. However, as explained concerning the difference between co-creation and co-operation in 1.2, a common goal is not required in an initial phase of co-creation as Star criticized [12]. Furthermore, intention as converged contexts among stakeholders in different contexts should be formed instead as a result of co-creation under enabling conditions such as autonomy, requisite variety, redundancy, fluctuation and creative chaos. Converged contexts lead co-creativity defined as a shared motivation to sustain co-creation (Sect. 4.2.3) and form intersubjectivity as a subjectivity of knowledge co-creation.

\subsection{Inverted vortex model}

The generalizable knowledge indicated in this study is the dynamic model IVM (Fig. 2), the model to form a dynamic relationship as a knowledge co-creating subjectivity among the organizations in various contexts. The phenomena observed during the case study have supported the IVM. In other words, the formation of autonomous knowledge-creating cluster among various stakeholders is observed as IVM represents the co-creation dynamics: knowledge co-creation formed intersubjectivity among organizations in various contexts and knowledge cocreation generated co-creativity, a shared motivation to sustain the co-creation.

IVM provides a systematic viewpoint on the separately explained relations between intersubjectivity and boundary object [18] with the IVM, which dynamically indicates the relationship between them driven by individual's context and also a higher resolution to ba where individual contexts and intersubjectivity are converged through cocreation, while Nonaka and Toyama's conceptual representation of ba explained that both the shared and individual contexts expand themselves through such interaction [22].

\subsection{Limitations}

The major limitation is that the knowledge co-creating subjectivity observed in this study was formed among horizontal relationship. The knowledge obtained from this study is not applicable to the formation process of different types of clusters, for example, the one in a vertical relationship, due to the differences in the knowledge-creating mechanism, except as a rationale to support expanding the scope of application to a hybrid type.

Another limitation to be considered is a cultural limitation regarding the capability to recognize and the matureness to deal with the value of undefined vagueness of co-creation, which is sometimes believed that the Western culture overlooks its value. However, this cultural limitation should be considered neither by country nor by industry just because the case introduced in this study is conducted in traditional Japanese industry. As having discussed above, the theoretically essential concepts structuring this co-creation model such as tacit knowledge (Sect. 1.2), intersubjectivity (Sect. 4.2.1), boundary object (Sect. 4.2.4) were advocated in West, and this IVM is practically developed with and supported by some of practitioners who form successful European Knowledge Innovation Cluster in information industry (Sect. 4.3).

\section{Conclusions}

In this paper, the authors focused on the formation process of horizontal knowledge-creating cluster generated among various contexts to answer the defined research question How can a dynamic relationship as a knowledge co-creating subjectivity be formed among the organizations in various contexts? In response to the question, the authors indicated the IVM with the case study on knowledge co-creating cluster formation as a practical application, where co-creation formed intersubjectivity among the organizations in various context, and it also generated co-creativity, a shared motivation among individuals to realize and develop their concepts.

How can we utilize this dynamic mechanism of co-creation for a desirable figure [33]: better business, a better society, or better well-being? In a connected society, various social infrastructures now have greater accessibility in a global scale for individuals and/or organizations that used to be excluded from the innovation ecosystem. This remarkable increase in accessibility to these social infrastructures leads to the horizontal fluidization of relationships. Compared to a vertical system with a clear goal decided by the top and followed by those lower in its fixed hierarchy, a horizontal system with dynamic changes in relationships among stakeholders enables individuals and 
organizations to pursue relationship optimization to realize a desired innovation. This implies that the importance to form and iterate relationships have rapidly increased in comparison to times when the innovation ecosystem was vertically fixed. Forming an autonomous knowledge co-creating subjectivity is fundamentally required as the inevitable first phase to structure a new innovation ecosystem. Knowledge obtained from this study will serve in designing and managing an inclusive and horizontal innovation ecosystem.

Acknowledgements We would like to express our gratitude to every stakeholder who has realized this case project with expertise and passion based on professionalism. Funding was provided by Japan Society for the Promotion of Science (Grant Nos. 16K16309, 16H03015).

\section{Compliance with ethical standards}

Conflict of interest On behalf of all authors, the corresponding author states that there is no conflict of interest.

\section{References}

1. Alford J (2016) Co-production, interdependence and publicness: extending public service-dominant logic. Public Manag Rev 18:673-691. https://doi.org/10.1080/14719037.2015.11116 59

2. Amabile TM (1983) The social psychology of creativity: a componential conceptualization. J Pers Soc Psychol 45:357-376. https ://doi.org/10.1037/0022-3514.45.2.357

3. Banerjee S, Sharma AK (2015) Co-creation as a risk-sharing strategy for the development of innovative EUV lithography technology in the semiconductor industry. Technol Anal Strateg Manag 27:1097-1113. https://doi.org/10.1080/09537325.2015.1060311

4. Bartodziej CJ (2017) The concept industry 4.0. Springer, Berlin

5. Benjamin J, Ph D (2004) Beyond doer and done to: an intersubjective view of thirdness. Psychoanal Q 73:5-46

6. Bower M (2014) Developing open intersubjectivity: on the interpersonal shaping of experience. Phenomenol Cogn Sci 14:455-474. https://doi.org/10.1007/s11097-014-9346-2

7. Csikszentmihalyi M (1998) Implications of a systems perspective for the study of creativity. In: Sternberg RJ (ed) Handbook of creativity. Cambridge University Press, Cambridge, pp 313-336

8. de Silva M, Wright M (2016) Ecosystem centric co-creation : a framework and typology. In: Proceedings of international conference of university industry interaction network (UIIN2017)

9. Donohoe J (2016) Husserl on ethics and intersubjectivity: from static and genetic phenomenology. University of Toronto Press, Toronto

10. Jokubauskiene R, Patasiene I, Bakanove A, Patasius M (2014) Model for evaluation of co-creation possibilities in the enterprise of knowledge - intensive business services. Soc Sci 1(83):7-16. https://doi.org/10.5755/j01.ss.83.1.6863

11. Konno N (2014) An introduction to "purpose engineering": an essay on "practical wisdom" and innovation

12. Leigh Star $S$ (2010) This is not a boundary object: reflections on the origin of a concept. Sci Technol Hum Values 35:601-617. https://doi.org/10.1177/0162243910377624
13. Matsumae A (2015) Application of design thinking to generate first-person initiative development in Arita: focusing on derived effects of co-creation process. In: Proceedings of 2015 EAROPH regional seminar in Japan. Eastern Regional Organization for Planning \& Human Settlements, Saga, Japan, pp 134-136

14. Matsumae A, Burrow K (2016) Business model generation canvas as a method to develop customer-oriented service innovation. In: Takashi M, Yuriko S, Hara T (eds) Serviceology for designing the future. Springer, Tokyo, pp 551-565

15. Matsumae A, Nagai $Y$ (2017) Formation of inter-subjectivity as a basis of sustainable collaborative innovation. Springer, Singapore

16. Matsumae A, Nagai $Y$ (2018) The function of co-creation in dynamic mechanism of intersubjectivity formation among individuals. In: Proceedings of the international design conference, design innovation, pp 1925-1936. https://doi.org/10.21278/ idc. 2018.0141

17. Nonaka I (1988) Creating organizational order out of chaos: selfrenewal in japanese firms. Calif Manag Rev 30:57-73. https://doi. org/10.2307/41166514

18. Nonaka I, Konno N (2012) The grammar of knowledge creating management for prudent capitalism. TOYO KEIZAI INC., Tokyo. ISBN13: 978-4492521991

19. Nonaka I, Takeuchi H (1995) The knowledge-creating: how Japanese companies create the dynamics of innovation. Oxford University Press, Oxford

20. Nonaka I, Toyama R (2003) The knowledge-creating theory revisited: knowledge creation as a synthesizing process. Knowl Manag Res Pract 1:2-10. https://doi.org/10.1057/palgrave. kmrp.8500001

21. Nonaka I, Von Krogh G, Voelpel S (2006) Organizational knowledge creation theory: evolutionary paths and future advances. Organ Stud 27:1179-1208. https://doi.org/10.1177/0170840606 066312

22. Nonaka I, Toyama R, Hirata T (2015) Managing flow: a process theory of the knowledge-based firm. Palgrave Macmillan, New york

23. Plucker JA, Dow GT (2004) Why isn't creativity more important to educational psychologists? Potentials, pitfalls, and future directions in creativity research. Educ Psychol 39:83-96. https://doi. org/10.1207/s15326985ep3902

24. Ramaswamy V, Ozcan K (2018) What is co-creation? An interactional creation framework and its implications for value creation. J Bus Res 84:196-205. https://doi.org/10.1016/j.jbusr es.2017.11.027

25. Reid SE, De Brentani U (2004) The fuzzy front end of new product development for discontinuous innovations: a theoretical model. J Product Innov Manag 21:170-184

26. Reynolds P, Miller B (1992) New firm gestation: conception, birth, and implications for research. J Bus Ventur 7:405-417. https:// doi.org/10.1016/0883-9026(92)90016-K

27. Sanders EB, Stappers PJ (2008) Co-creation and the new landscapes of design. CoDesign 4:5-18. https://doi. org/10.1080/15710880701875068

28. Sawyer RK (2011) Explaining creativity: the science of human innovation, 2nd edn. Oxford University Press, New York

29. Shostack GL (1982) How to design a service. Eur J Mark 16:49-63. https://doi.org/10.1108/EUM0000000004799

30. Skaržauskaitè M (2013) Measuring and managing value cocreation process: overview of existing theoretical models. Soc Technol 3:115-129. https://doi.org/10.13165/ST-13-3-1-08

31. Star SL, Griesemer JR (1989) Institutional ecology, 'translations' and boundary objects: amateurs and professionals in Berkley's museum of vertebrate zoology. J Soc Stud Sci 19:387-420 
32. Tari Kasnakoglu B (2016) Antecedents and consequences of cocreation in credence-based service contexts. Serv Ind J 36:1-20. https://doi.org/10.1080/02642069.2016.1138472

33. Taura T, Nagai $Y$ (2009) A definition of design and its creative features. Proc Int Assoc Soc Des Res 2009:1-10

34. Von Krogh G, Ichijo K, Nonaka I (2000) Enabling knowledge creation: how to unlock the mystery of tacit knowledge and release the power of innovation. Oxford University Press, Oxford
35. Zahavi D (2001) Husserl and transcendental intersubjectivity. Ohio University Press, Ohio

Publisher's Note Springer Nature remains neutral with regard to jurisdictional claims in published maps and institutional affiliations. 